\title{
Sleep and daytime sleepiness in upper airway resistance syndrome compared to obstructive sleep apnoea syndrome
}

\author{
C. Guilleminault*, Y. Do Kim, S. Chowdhuri*, M. Horita", M. Ohayon*, C. Kushida*
}

Sleep and daytime sleepiness in upper airway resistance syndrome compared to obstructive sleep apnoea syndrome. C. Guilleminault, Y. Do Kim, S. Chowdhuri, M. Horita, M. Ohayon, C. Kushida. (C) ERS Journals Ltd 2001.

ABSTRACT: This study has investigated differences in the nocturnal sleep and daytime sleepiness among patients with obstructive sleep apnoea syndrome (OSAS), upper airway resistance (UARS), sleep hypopnoea syndrome, and normal control subjects, using sleep scoring and spectral activity analysis of the electroencephalogram (EEG).

Twelve nonobese males with UARS aged 30-60 yrs were recruited. These subjects were strictly matched for age and body mass index with twelve OSAS patients, 12 sleep hypopnoea syndrome patients, and 12 normal controls, all male. Daytime sleepiness was evaluated using the Epworth Sleepiness Scale (ESS) and the Multiple Sleep Latency Test (MSLT). The macrostructure of sleep was determined using international criteria and spectral analysis of the sleep EEG was obtained from a central lead.

The sleep macrostructure of OSAS and UARS patients was significantly different from that of controls. These patients were also sleepier during the daytime than controls. Complaints of tiredness and daytime sleepiness, ESS and MSLT scores were similar in the different patient groups. Mild dysmorphia was present in all three patient groups. However, nocturnal sleep was significantly different among the different groups. OSAS patients had significantly more awake time during sleep than the UARS patients. The spectral activity of the total sleep time of the patient groups also differed significantly from that of controls. When the sleep spectral activity of UARS and OSAS patients were compared, OSAS patients had less slow wave sleep activity than UARS patients. UARS patients had a significantly higher absolute power in the 7-9 $\mathrm{Hz}$ bandwidth than OSAS patients. The absolute delta power over the different sleep cycles was also different between controls and patients, and between UARS and OSAS patients.

There are clear differences in the macrostructure and spectral activity of sleep between upper airway resistance and obstructive sleep apnoea syndrome patients, demonstrated by differences in the cortical activity recorded in the central lead during sleep. Despite these nocturnal sleep differences, the tests of subjective daytime sleepiness are not significantly different.

Eur Respir J 2001; 17: 838-847.
*Stanford University Sleep Disorders Clinic and Research Center, Stanford, USA, " Dept of Neuropsychology, Toyama Medical and Pharmaceutical University, Toyama, Japan and "Dept of Electronics and Information Engineering, Isikawa National College of Technology, Ishikawaka-ken, Japan.

Correspondence: C. Guilleminault, Stanford Sleep Center, 401 Quarry Road, Suite 3301-A, Stanford, CA 94305 , USA.

Fax: 16507258910

Keywords: Alpha electroencephalogram arousal

daytime sleepiness

microstructure of sleep

obstructive sleep apnoea syndrome

patients

upper airway resistance

Received: May 252000

Accepted after revision January 42001

C. Guilleminault was supported by an Academic Award from the Sleep Center from the National Institute for Heart, Blood, and Lung of the National Institute for Health. This presentation was part of a dissertation for a doctorate in Biology from the University of Grenoble (France).
Patients with obstructive sleep apnoea syndrome (OSAS) and upper airway resistance syndrome (UARS) both complain of daytime tiredness, fatigue, and variable degrees of sleepiness [1, 2]. Nocturnal polygraphic recordings, however, indicate differences between these two groups. During apnoeic or hypopnoeic events of $\geqslant 10 \mathrm{~s}$, OSAS and obstructive sleep hypopnoea syndrome (OSHS) patients show a substantial decrease in oxygen saturation $\left(\mathrm{S}_{\mathrm{a}}, \mathrm{O}_{2}\right)$. Conversely, in UARS, $\mathrm{Sa}, \mathrm{O}_{2}$ may fluctuate somewhat but stays $>90 \%$; the abnormal events are short, involving one or two breaths and consist of flow limitation but no apnoea $[1,3]$.

The aim of this study was to investigate the degree of daytime sleepiness and the characteristics of the sleep electroencephalogram (EEG) by examining the macroand microstructure of sleep in addition to the spectral activity recorded in the central EEG leads during sleep. The latter was performed using spectral analysis in rigorously matched OSAS, UARS, and normal subjects. It was questioned whether the basic dissimilarity in the upper airway closure pattern in UARS versus OSAS subjects could lead to differences in their sleep EEG. Not enough subjects with sleep hypopnoea syndrome [4], who met the strict matching requirements, could be found, therefore, the data obtained on sleep hypopnoea subjects is only presented for completeness. The analysis will mainly focus on the three strictly matched subject groups: OSAS patients, UARS patients, and normal controls.

\section{Methods}

\section{Subject selection}

Twelve male UARS patients were recruited from the sleep disorder clinic. They were representative of the 


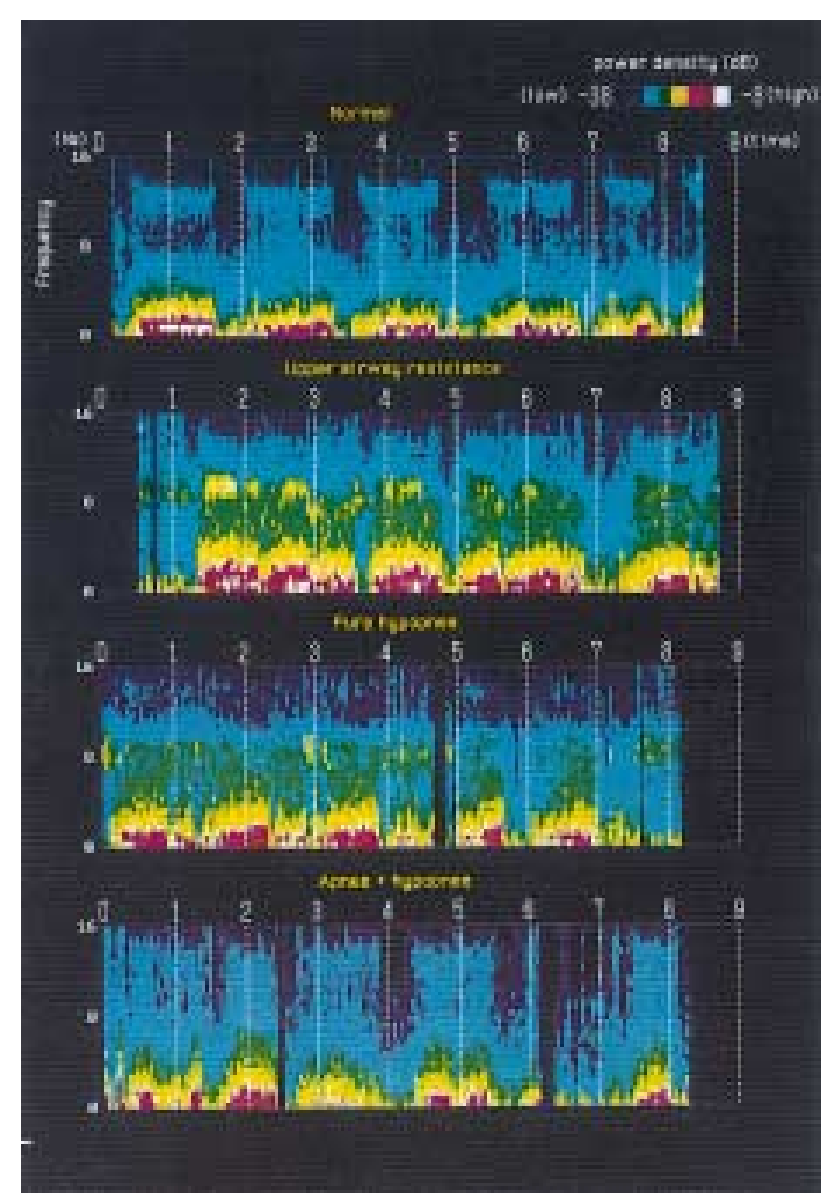

Fig. 1. - Spectral analysis results of the sleep electroencephalogram (EEG) obtained from a central lead in four sex-, age-, and body mass index-matched subjects: normal control; upper airway resistance patient; hypopnoea syndrome patient; and an obstructive sleep apnoea syndrome patient. EEG frequency $(0.5-16 \mathrm{~Hz})$ is plotted against time in $\mathrm{h}$ of nocturnal sleep. The colours represent the strength of the power density of each frequency from black (lowest) to white (highest) as indicated in the top-right corner of the figure. Note the higher absolute power density in $7-9 \mathrm{~Hz}$ bandwidth in upper airwat resistance compared with others.

patients diagnosed with UARS in the clinic in terms of type of complaint and nocturnal polysomnographic findings. Patients were $30-60$ yrs of age, with a body mass index (BMI) of $24-27.5 \mathrm{~kg} \cdot \mathrm{m}^{-2}$, and with $>10$ respiratory-related arousals ( $\geqslant 3 \mathrm{~s}$ duration) per $\mathrm{h}$ of sleep. Each of these subjects were then matched by sex, BMI, and age with an OSAS patient, an OSHS patient, and a normal control. The tolerated variance for BMI and for age was fixed prior to recruitment at $\pm 0.5 \mathrm{~kg} \cdot \mathrm{m}^{-2}$ and $\pm 1 \mathrm{yr}$, respectively. All noncontrol subjects had clinical complaints of daytime tiredness, fatigue and/or daytime sleepiness. Patients had scores of 3-5 in the Sleep Disorders Questionairre (SDQ) [5] on daytime sleepiness questions, (the SDQ uses a scale from $0=$ "never" to $5=$ "always"), and Epworth Sleepiness Scale (ESS) scores >9 [6]. Control subjects had normal polysomnograms and no clinical complaints of a sleep disorder.

Normal controls were recruited by advertisement from the community. After clinical screening, a total of 30 nocturnal polysomnograms were performed to

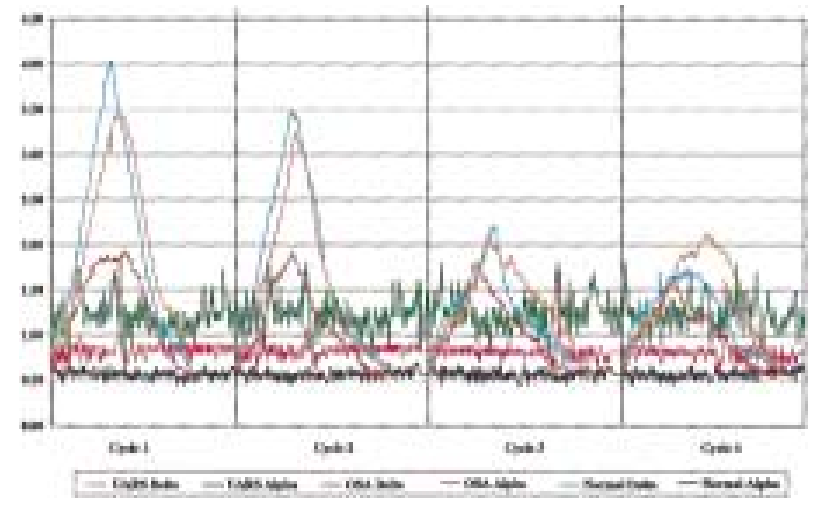

Fig. 2. - Mean absolute power for delta and alpha bands for normal controls, obstructive sleep apnoea syndrome (OSAS) and upper airway resistance (UARS) patients during one night. During the different sleep cycles, the normal evolution of slow wave activity (SWA) in control subjects can be seen with a progressive decrease from the beginning to the end of the night. UARS patients have a significantly higher SWA in cycles $2-4$, and more so at the end of the night than normal controls, and SWA is significantly decreased in all cycles in OSAS patients, compared to both normal controls and UARS patients. Mean alpha activity is increased throughout the night in UARS patients. This increase is significantly more important compared not only to normal controls but also to OSAS subjects.

obtain the 12 matched control subjects. The matched OSAS and OSHS patients were selected from a database by a computer program. Two-hundred and seventy-six OSAS patients were reviewed to obtain the 12 matched subjects. Searching the current database of $>1,000$ sleep disordered breathing patients seen during the same period of time, only two patients could be matched with OSHS as defined by Gould et al [4]. In order to recruit a further 10 subjects corresponding to the above definition [4], the tolerated variance was broadened to $\pm 5 \mathrm{~kg} \cdot \mathrm{m}^{-2}$ for BMI and \pm 5 yrs for age. In view of the change in matching criteria, and the impact that obesity might have on the microstructure of sleep, the data obtained on OSHS are given only for completeness. Presentation of any raw data (fig. 1) always involves subjects who were matched as planned.

\section{Protocol}

Definition of the population studied. Diagnostic workup. All subjects completed a validated SDQ [5] covering sleep and its disorders and the ESS [6]. They all underwent clinical interview, clinical evaluation and diagnostic nocturnal polygraphic recording, which included monitoring of EEG (C3/A2, C4/A1 from the $10 / 20$ international electrode placement system), electrooculogram, chin and leg electromyogram and electrocardiogram (modified $\mathrm{V}_{2}$ lead). Respiration was measured by means of airflow (nasobuccal thermocouple), noncalibrated inductive respiratory plethysmography, oesophageal pressure $\left(P_{\text {oes }}\right)$ recording and pulse oximetry.

The clinical evaluation involved examination of the upper airway. Maxillo-mandibular evaluation and evaluation of nasopharynx were performed by visual inspection according to the formula published by 
KusHidA et al. [7]. Tonsils were evaluated on a subjective scale, from $0=$ "absent" to $4=$ "kissing tonsils"; the size and position of the uvula were evaluated on a scale from 1 to $4 \quad(1=$ "thin and elongated uvula", $4=$ "large uvula located well behind the base of the tongue"); the presence/absence of deviated nasal septum was assessed; and nasal turbinates were scored at rhinoscopic examination, from $1=$ "normal" to $3=$ "clearly enlarged". To be considered as a UARS patient, subjects must have had a complaint of daytime sleepiness and abnormal scores on SDQ and ESS, and an apnoea/hypopnoea index (AHI) $<5$ events $\cdot \mathrm{h}^{-1}$ of sleep; the lowest $S \mathrm{a}, \mathrm{O}_{2}$ during the total polysomnogram recording must not have been $<90 \%$. In addition, evaluation of the oesophageal manometry curve and other variables of the nocturnal polysomnogram must have shown features previously described [1, 2], i.e. presence of "crescendos" with progressively more negative peak end-inspiratory $P_{\text {oes}}$, terminated by a return toward less negative pressure (called a "Poes reversal") and associated with an EEG arousal of $\geqslant 3 \mathrm{~s}$; or an abnormal effort with $P$ oes reversal but no crescendos. UARS subjects must have presented $\geqslant 10$ respiratory-related arousals per $h$ of sleep, of $\geqslant 3 \mathrm{~s}$, not corresponding to the definition of "apnoea" and "hypopnoea", in this first, diagnostic recording to be included in the study. In summary, the clinical presentation and polygraphic recording of the UARS patients was similar to the one reported in the initial description of the syndrome [1]. The subdivision into OSAS and OSHS was based on polysomnographic results. The definitions used to score "apnoea" and "hypopnoea" are given below. Subjects were labelled as OSAS if $\geqslant 80 \%$ of the abnormal breathing events had been scored as "apnoeas", whereas OSHS was attributed if $\geqslant 80 \%$ of the events were scored as "hypopnoeas".

The research polygraphic recording. Once identified for the study, subjects signed informed consent forms. They were then asked to be in the laboratory by 19:00 h, where they underwent a second evaluation that included a nocturnal polysomnographic recording of at least $8.5 \mathrm{~h}$ in the supine position, with verification of position by video camera and body sensors placed on the chest. The supine position was kept by placing long inflatable pillows on each side of the patient. The air pumped into each pillow comfortably maintained the supine position and avoided sleep disturbances. Selection of an obligatory supine position was made after checking that this position had been the predominant one during the first recording; it was selected to avoid interference of body position as an uncontrolled variable. The same variables listed above and monitored during the diagnostic polysomnogram were monitored again during the research polygraphic recording. But the inductive respiratory plethysmography was calibrated at the beginning of the night for this second recording. To perform appropriate spectral analysis, the central EEG derivations $\mathrm{C} 3 / \mathrm{A} 1$ and $\mathrm{C} 4 /$ $\mathrm{A} 2$ were monitored in addition to the recommended C3/A2 and C4/A1 [9]. The nocturnal recording was followed by a multiple sleep latency test (MSLT) that included five naps scheduled every $2 \mathrm{~h}$ starting at 09:00 $\mathrm{h}$ [8]. None of the subjects had received any treatment for their sleep disordered breathing at the time of the study.

Scoring criteria and data analysis of research recordings. Scoring of sleep and breathing. Polygraphic recordings were scored by 30 -s epochs, following the international criteria outlined by RECHTSCHAFFEN and KALES [9], and micro-arousals were determined following the American Sleep Disorders Association (ASDA) criteria [10]. Visual recognition of EEG changes in the central EEG leads is dependent on the background EEG. Depending on the background, visual scoring using short duration has a high interscorer variability. The international criteria request a minimum of $16 \mathrm{~s}$ of EEG changes before scoring an "arousal". A $16 \mathrm{~s}$ arousal indicates a long cortical change that will involve all parts of the cortex. It was determined that an "awakening" occurred when there was a change of the visually scored EEG lasting $>30 \mathrm{~s}$, using RECHTSCHAFFEN and KALES' [9] criteria. "Awakenings" were identified in all records independently of subject group. The $30 \mathrm{~s}$ "awakening" measurement was introduced to flag visually scored arousals associated with longer wake periods. This scoring had two advantages: to recognize the frequency of longer arousals and to help in the preparation of the records to be submitted to spectral analysis of the sleep EEG. For appropriate analysis, all 5-s epochs of "wake" have to be deleted prior to mathematical treatment. Arousals lasting $\geqslant 30 \mathrm{~s}$ are easy to identify visually, as their EEG always include beta activity. The segments labelled as "awakenings" can be quickly recognized and dealt with when preparing records for spectrum analysis of the sleep EEG. Arousals of shorter duration were carefully looked at thereafter. Wake after sleep-onset was derived from the determination of all arousals lasting $\geqslant 3 \mathrm{~s}$, using ASDA and RECHTCHAFFEN and KALES' [9] scoring criteria. Recognition of short arousals can be a challenge and a lot of attention was given to the identification of these shorter arousals.

The same equipment (including thermocouples) was used on all subjects and the same scorer, blind to clinical symptoms and subject classification, performed the initial apnoea and hypopnoea scoring.

As the definitions of apnoea and hypopnoea are critical for the results of the investigation, the scoring criteria were clearly outlined before the study. Apnoeas were scored as per the international criteria [11]. The following criteria were used to score hypopnoeas: oronasal flow amplitude change, $\mathrm{Sa}, \mathrm{O}_{2}$ change and EEG change. A hypopnoea was scored when the amplitude of the thermocouple signal changed by $\geqslant 50 \%$ but $\leqslant 70 \%$ and was associated with either a decrease in $\mathrm{Sa}, \mathrm{O}_{2}$ of $\geqslant 3 \%$ compared to the immediately preceding epoch, or a visually scored EEG arousal at the end of the event. The EEG arousal could be as short as $3 \mathrm{~s}$ [10]. The event must have lasted $\geqslant 10 \mathrm{~s}$. Recordings were scored on a computer, and the $P_{\text {oes }}$ signal was blanked out during the initial scoring of apnoeas and hypopnoeas. The scorer identified all the 
abnormal breathing events, and a second scorer then reassessed the scoring. The second scorer, who remained the same throughout the study, was also blind to clinical symptoms and subject classification. The second scoring was not a full rescoring, but a review of the first scorer's identification of apnoeas and hypopnoeas. If the second scorer questioned the identification of an event, a third expert, also blind to the status of the subject, examined the study. The three scorers evaluated each questioned event in a joint session. Classification of an event was accepted when at least two of the three scorers concurred. Six-hundred and ninety-four events $(6.6 \%$ of the total number of events) were questioned. All records were then rescored with the addition of the $P_{\text {oes }}$ signal. The prior identification of apnoeas and hypopnoeas was deleted from the recording during this rescoring. There was a minimum of 6 weeks and a maximum of 4 months before the same record was rescored by the initial scorer. This scorer was asked again to identify apnoeas and hypopnoeas. In addition, they were asked to identify "abnormal breathing events" that were associated with abnormal respiratory effort but that did not fit the definition of "hypopnoeas". These events were defined based on the analysis of all recorded channels, including $P_{\text {oes }}$ and were called "Poes events". One $P_{\text {oes }}$ pattern, demonstrating a progressively more negative peak end-inspiratory pressure with each successive breath, was called a "crescendo". During a "crescendo", there was no clear change in the thermocouple readings i.e. no change meeting the definition of a hypopnoea, and the event was terminated by an abrupt drop in respiratory effort (called a $P_{\text {oes }}$ reversal) with a short $3-15$ s EEG arousal, with or without a burst of chin electromyogram (EMG) activity [10]. Another pattern involved the presence of persistently increased respiratory effort for several breaths and without the progressively increasing effort as seen in a "crescendo" [3]. This pattern was also terminated by a $P_{\text {oes reversal }}$ with a short 3-15 s EEG arousal, with or without burst of chin EMG. Finally "crescendos" without arousal lasting $\geqslant 3 \mathrm{~s}$ but associated with a $P$ oes reversal at the end of the event were also included in the analysis. For the event to be scored a Poes event, any change in the thermocouple tracing had to be noted in only the one or two breaths preceding the $P$ oes reversal, and the change could not exceed the lowest decrease required for the scoring of a hypopnoea. The recognition of "abnormal breathing event" associated with the scoring of short arousal led to the determination of a Respiratory Disturbance Index (RDI) different from the AHI. The same rescoring of the records as described for the scoring of apnoeas and hypopnoeas was applied to the scoring of $P_{\text {oes }}$ events. This scoring method allowed identification, without ambiguity, of patients with OSAS, UARS, and OSHS

Electroencephalogram spectral analysis. EEGs were low-pass filtered and digitized on-line at a sampling rate of $128 \mathrm{~Hz}$. The central lead (C3/A1) was selected as the sampling site in all subjects. Spectral analysis was performed by fast Fourier transformation for 5-s mini-epochs using a Hamming window. Each mini-epoch was visually inspected to identify artefacts. Since records were to be submitted to spectrum analysis of the EEG, attention was paid during data collection to avoid artefacts that would affect good analysis. If artefacts, however, were present, the mini-epoch was rejected and scored as missing data to preserve sleep continuity. Six consecutive 5-s epochs were then smoothed and averaged to give a 30 -s epoch corresponding to the 30 -s epochs of sleep scoring.

Frequency bands were defined. Due to the risk of artefacts, the $0-0.5 \mathrm{~Hz}$ frequency band was not considered in the analysis. The analysis was performed using the definition of five frequency bandwidths: delta $(0.75-3.75 \mathrm{~Hz})$, theta $(4.0-7.75 \mathrm{~Hz})$, alpha $(8-12 \mathrm{~Hz})$, sigma $(12.25-15 \mathrm{~Hz})$ and beta $(\geqslant 15.25 \mathrm{~Hz})$. The delta frequency was also analysed in low $(0.5-2 \mathrm{~Hz})$ and high $(2-4 \mathrm{~Hz})$ delta bands and a $1-\mathrm{Hz}$ band subanalysis was performed on high theta $(7-8 \mathrm{~Hz})$ and alpha frequencies $(8-12 \mathrm{~Hz})$. The rationale for this substudy was that "arousal" was considered to involve a

Table 1. - Clinical variables in the four subject groups

\begin{tabular}{|c|c|c|c|c|c|c|c|c|c|c|c|c|}
\hline \multirow{2}{*}{$\begin{array}{l}\text { Subject } \\
\text { no. }\end{array}$} & \multicolumn{4}{|c|}{ Age yrs } & \multicolumn{4}{|c|}{$\mathrm{BMI} \mathrm{kg} \cdot \mathrm{M}^{-2}$} & \multicolumn{4}{|c|}{ ESS SCORE } \\
\hline & Controls & UARS & OSAS & OSHS & Controls & UARS & OSAS & OSHS & Controls & UARS & OSAS & OSHS \\
\hline 1 & 30.3 & 30.1 & 30.4 & 30 & 24 & 24.1 & 23.8 & 24.4 & 3 & 12 & 11 & 11 \\
\hline 2 & 41.1 & 41.2 & 39.9 & 43.9 & 26 & 26.1 & 26.2 & 27.3 & 5 & 11 & 13 & 12 \\
\hline 3 & 54.2 & 54 & 54.3 & 51.9 & 27 & 27 & 27.3 & 28.9 & 7 & 12 & 14 & 13 \\
\hline 4 & 37.1 & 37.3 & 37.5 & 39.9 & 24.4 & 24.6 & 24.7 & 26 & 4 & 12 & 12 & 12 \\
\hline 5 & 42.5 & 42.4 & 42.1 & 42 & 24.2 & 24.3 & 24.6 & 24.2 & 5 & 12 & 13 & 11 \\
\hline 6 & 38.4 & 38.9 & 38.9 & 42.3 & 23.6 & 23.9 & 24.1 & 28 & 3 & 11 & 12 & 14 \\
\hline 7 & 44.6 & 44.5 & 44.5 & 48.3 & 25.2 & 24.8 & 25.3 & 27 & 4 & 13 & 12 & 13 \\
\hline 8 & 46.1 & 46.3 & 46.3 & 41.2 & 26.4 & 26.6 & 26.9 & 25.1 & 2 & 12 & 13 & 12 \\
\hline 9 & 36.8 & 36.6 & 36.6 & 39.9 & 24.1 & 24.5 & 24.3 & 26.7 & 0 & 11 & 12 & 12 \\
\hline 10 & 50.2 & 50.6 & 50.6 & 53.5 & 25.6 & 25.2 & 25.5 & 26.9 & 7 & 13 & 14 & 14 \\
\hline 11 & 33.8 & 33.9 & 33.9 & 36.7 & 22.8 & 23.1 & 23.4 & 27 & 2 & 11 & 11 & 13 \\
\hline 12 & 48.1 & 48.6 & 48.6 & 52.1 & 24.3 & 24.5 & 24.7 & 28.5 & 4 & 13 & 12 & 14 \\
\hline Mean & 41.9 & 42.0 & 42.0 & 43.5 & 24.8 & 24.9 & 25.0 & 26.7 & 3.8 & 11.9 & 12.4 & 12.6 \\
\hline SD & 7.05 & 7.10 & 7.12 & 6.95 & 1.24 & 1.15 & 1.22 & 1.51 & 2.04 & 0.79 & 1.00 & 1.08 \\
\hline
\end{tabular}

Data presented as absolute values; BMI: body mass index; ESS: Epworth Sleepiness Scale; UARS: upper airway resistance; OSAS: obstructive sleep apnoea syndrome; OSHS: obstructive sleep hypopnoea syndrome. 
switch to high theta and alpha rhythm. The power spectra were expressed in absolute values. The power value for a $50 \mu \mathrm{V}$ calibrated signal input was zero decibel, and this value was used to calculate the power density of each band. The sleep cycles were defined according to the FEINBERG and FLOYD [12] criteria, as a succession of nonrapid eye movement (NREM) sleep episodes lasting $\geqslant 15 \mathrm{~min}$ followed by an REM sleep episode of $\geqslant 5 \mathrm{~min}$. An NREM sleep episode was defined as the time interval between the first occurrence of stage 1 NREM sleep and the first occurrence of REM sleep within a cycle. Each of the 5 -s epoch average obtained every $30 \mathrm{~s}$ was placed in an NREM and REM sleep cycle in temporal succession. In order to submit to statistical analysis, each sleep cycle was subdivided into 5-min segments and during these segments mean \pm SD were obtained for each of the considered EEG bands. These 5-min averages were submitted to statistical analyses.

\section{Statistical analysis}

Kruskal-Wallis one way analysis of variance (ANOVA) was first performed on the nonsleep-EEG group data. A Bonferroni post hoc comparison following the nonparametric Kruskal-Wallis ANOVA was performed for comparison between groups. For power spectra comparison, the following was carried out. For each band the mean value obtained for each group was determined for each data entry. There were 200-220 data entry per sleep cycle. The first comparisons were performed comparing the sum of each band in a patient for each cycle. This first analysis allowed determination of whether the sum of one EEG band tracing obtained for a given cycle differs between the groups. Thereafter, for each EEG band ANOVA with post hoc Bonferroni and Scheffe tests were performed. If there was nonhomogeneity of variance between groups, a nonparametric test (Kruskal-Wallis) was also calculated to confirm that the significant differences noted with the analysis of variance were also significant using a nonparametric test. The Systat version 7.0 (Systat, Evanston, IL, USA) and the Statistical Package for Social Sciences (SPSS) version 9.0 (SPSS, Chicago, IL, USA) statistical packages were used for the statistical analyses.

\section{Results}

\section{Clinical evaluation}

The maxillo-mandibular evaluation using the KusHIDA et al. [7] criteria yielded a score of 90 . None of the patient group scores were significantly different from one another. This indicates that all patients had mild facial dysmorphia.

\section{Daytime sleepiness}

Tables 1 and 2 show demographic information and the results of nocturnal respiratory variables obtained

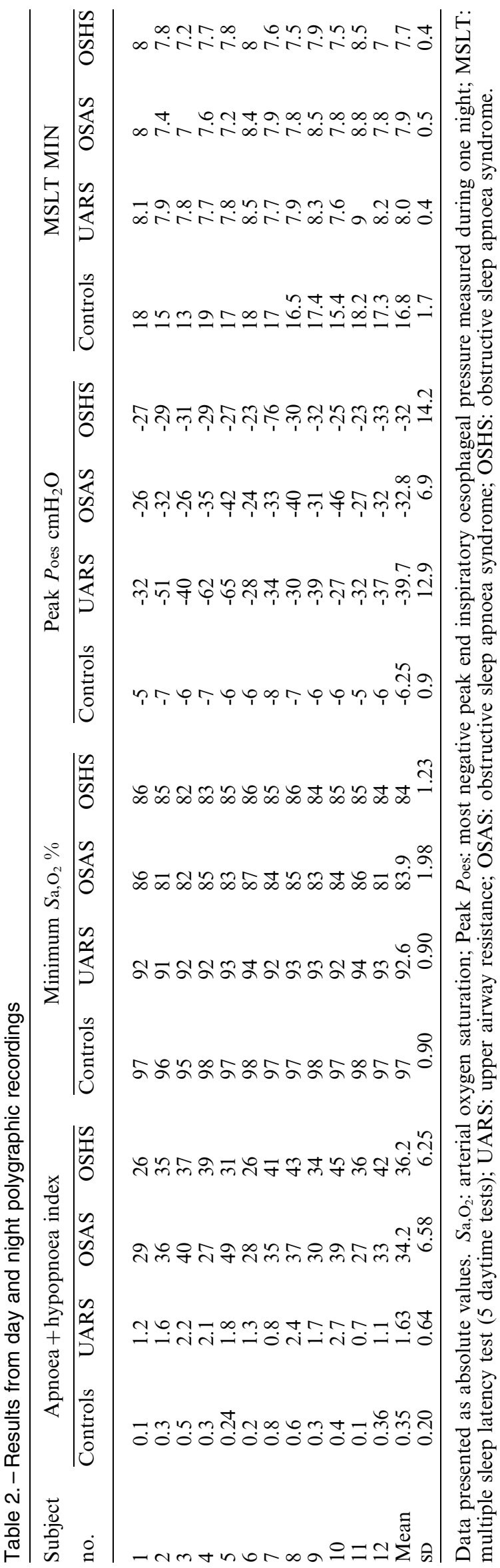


Table 3. - Results of nocturnal sleep scoring

\begin{tabular}{lcccc}
\hline & Control & UARS & OSAS & OSHS \\
\hline TST min & $454 \pm 24$ & $383 \pm 30$ & $317 \pm 38$ & $341 \pm 49$ \\
Stage 1 NREM sleep min & $30 \pm 6(6.6)$ & $70 \pm 17(18.3)$ & $71 \pm 22(29)$ & $73 \pm 25(21.4)$ \\
Stage 2 NREM sleep min & $238 \pm 21(52.4)$ & $166 \pm 28(43.3)$ & $170 \pm 36(53.6)$ & $190 \pm 41(52.7)$ \\
Stage 3 - 4 NREM sleep min & $98 \pm 11(21.5)$ & $47 \pm 11(12.3)$ & $30 \pm 13(9.5)$ & $38 \pm 12(11.1)$ \\
REM sleep min & $87 \pm 9(19)$ & $60 \pm 12.6(16)$ & $46 \pm 11(14.5)$ & $50 \pm 12.5(14.6)$ \\
WASO min & $12 \pm 8$ & $67 \pm 23$ & $133 \pm 27$ & $124 \pm 26$ \\
\hline
\end{tabular}

Data expressed as mean \pm SD $(\%)$. UARS: upper airway resistance; OSAS: obstructive sleep apnoea syndrome; OSHS: obstructive sleep hypopnoea syndrome; TST: total sleep time; REM: rapid eye movement; NREM: non-REM; WASO: wake after sleep onset.

from the polygraphic recording and MSLT for each of the 48 subjects in the study. As can be seen, three of the four groups (controls, UARS, OSAS) were very closely matched for age and BMI. ANOVA confirmed this point. Regarding subjective (ESS, table 1) and objective (MSLT, table 2) sleepiness, there was an obvious difference between the scores of patients and normal controls $(\mathrm{p}<0.00001)$. ESS and MSLT results, however, were similar in the patient groups. The sleepiness indices indicated greater sleepiness in each of the patient groups than in controls, despite the variance in the lowest $S_{\mathrm{a}}, \mathrm{O}_{2}$ in the different patient groups.

\section{Nocturnal polysomnography}

Macrostructure of sleep. Total time in bed was a minimum of $518 \mathrm{~min}$ and maximum of $547 \mathrm{~min}$. The mean total sleep time calculated using RECHTSCHAFFEN and KALES' [9] criteria (table 3) was significantly higher in controls than in all patient groups $(p=0.01)$. OSAS patients had significantly shorter total sleep time than UARS patients $(\mathrm{p}=0.01)$, this was related to a higher amount of wake time with a higher number of $30-\mathrm{s}$ epochs, scored as "awake".

Stages 1 and 2 NREM sleep were not significantly different between the three patient groups, despite the fact that control subjects had significantly less stage 1

Table 4.-Respiratory Disturbance Index in the four groups of subjects

\begin{tabular}{lcccc}
\hline Subject no. & Controls & UARS & OSAS & OSHS \\
\hline 1 & 1.1 & 35.2 & 33.0 & 28.0 \\
2 & 1.4 & 44.6 & 40.0 & 38.0 \\
3 & 1.9 & 42.2 & 42.1 & 40.0 \\
4 & 1.1 & 35.1 & 32.2 & 42.1 \\
5 & 1.3 & 47.8 & 51.3 & 34.1 \\
6 & 1.8 & 33.3 & 34.4 & 29.0 \\
7 & 2.0 & 37.8 & 36.2 & 43.0 \\
8 & 1.6 & 43.4 & 40.0 & 44.8 \\
9 & 1.3 & 31.7 & 32.1 & 37.2 \\
10 & 1.7 & 42.7 & 41.8 & 47.4 \\
11 & 0.6 & 33.3 & 33.7 & 38.2 \\
12 & 1.6 & 35.1 & 34.9 & 43.6 \\
Mean & 1.5 & 38.5 & 37.6 & 38.8 \\
SD & 0.4 & 5.3 & 5.7 & 6.0 \\
\hline
\end{tabular}

Data presented as absolute values. UARS: upper airway resistance; OSAS: obstructive sleep apnoea syndrome; OSHS: obstructive sleep hypopnoea syndrome.
NREM sleep than patients $(\mathrm{p}=0.001)$. The percentage of time spent in slow wave sleep (stage 3-4 NREM sleep) and in REM sleep was significantly higher $(p=0.001)$ in normals compared to patients, regardless of group. In addition, the total time spent in stages 3-4 NREM sleep was significantly higher $(p=0.01)$ in UARS patients than in OSAS patients (table 3 ).

Other polygraphic variables are also presented in table 1. ANOVA showed that there were significant differences among the four groups for peak $P_{\text {oes, }}$ lowest

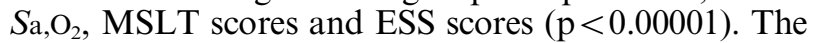
control group was significantly different $(\mathrm{p}<0.00001)$ from the OSAS and OSHS groups for the AHI, but was not different from the UARS group. The RDI, formed by the addition of the AHI and the nonapnoeic, nonhypopnoeic (as defined) other abnormal breathing events during sleep, were calculated. Results are presented in table 4. As can be seen, controls are significantly different from all other groups, $\mathrm{p}=0.0001$, but there is no significant difference among the different patients groups. The difference between AHI and RDI is, however, very important when OSAS and UARS are compared. The UARS group was also significantly different from the OSAS group for the lowest $\mathrm{Sa}_{\mathrm{a}} \mathrm{O}_{2}$ recorded $(\mathrm{p}<0.00001)$ as well as for $\mathrm{AHI}$ $(\mathrm{p}<0.00001)$, but was not significantly different with respect to peak Poes, MSLT, and ESS results. The OSAS group and OSHS group were not significantly different from each other for any of the considered variables.

\section{Analysis of power spectra during sleep}

Wakefulness epochs as defined by RECHTSCHAFFEN and KALES' [9] criteria (that were higher in the OSAS group) were scored as "missing data" in the analysis, as were epochs with artefacts obscuring EEG leads (mostly due to large body movements).

Nonrapid eye movement sleep. During NREM sleep, all patient groups had a decrease in slow wave activity (SWA) $(0-3.5 \mathrm{~Hz})$ compared to controls (ANOVA $\mathrm{p}=0.01)$. When analyses were performed for low and high delta bands, the decrease in the low $(0.5-2 \mathrm{~Hz})$ delta band was significant for both the UARS $(\mathrm{p}=0.01)$ and OSAS groups $(\mathrm{p}=0.001)$ compared to controls. The decrease in the high delta band was also significant for both of these groups compared to controls (UARS $\mathrm{p}=0.02$; OSAS $\mathrm{p}=0.01)$. Similarly, there was a 
significant decrease in the sigma band (ANOVA $\mathrm{p}=0.01$ ) in all patient groups compared to controls $(13-14 \mathrm{~Hz})$. Finally, controls had significantly less $(\mathrm{p}=0.001)$ beta activity $(\geqslant 16 \mathrm{~Hz})$ than the patient groups.

When compared individually to controls, UARS and OSAS patients had different findings in the theta and alpha bands. UARS patients had a significantly higher power density than controls in the alpha band (0.001) and OSAS patients were also higher $(\mathrm{p}=0.05)$ compared to controls. The subanalysis performed on the $7-8,8-9$ and other $1 \mathrm{~Hz}$ band increments to $11-12 \mathrm{~Hz}$, indicates that there was a significantly higher power density $(p=0.01)$ in the $7-8,8-9$ and $9-10 \mathrm{~Hz}$ bands in UARS. The results were different in OSAS patients, with a significant difference $(\mathrm{p}=0.05)$ only in the $9-10 \mathrm{~Hz}$ band.

When OSAS and UARS patients were compared to each other, the power density in the theta bandwidth was not significantly different, but UARS patients had a significantly higher power density $(p=0.01)$ in the $7-8 \mathrm{~Hz}$ bandwidth and in the $8-9 \mathrm{~Hz}$ bandwidth; the difference was nonsignificant in the $9-10 \mathrm{~Hz}$ band, despite a higher power density in UARS patients. Overall, UARS patients showed a clearly higher absolute power in the $7-10 \mathrm{~Hz}$ bandwidths than did OSAS patients (figs. 1 and 2). Interestingly, the $10-11 \mathrm{~Hz}$ and $11-12 \mathrm{~Hz}$ bands did not show these differences.

Rapid eye movement sleep. Normal controls were significantly different from all patient groups; the latter presented significantly lower SWA, (ANOVA $\mathrm{p}=0.05$ ). Low and high delta analysis showed that the decrease was particularly significant in the low delta band $(p=0.01)$. When UARS and OSAS were compared to controls independently, the same significant differences were found in the low delta range. When compared to each other, UARS had a higher delta power than the OSAS patients. The UARS patients also had a significantly higher power density than controls in the alpha range $(\mathrm{p}=0.01)$. OSAS patients had a nonsignificant increase in alpha power density compared to controls. When UARS and OSAS patients were compared to each other, there was a significantly higher power density in the $8-10 \mathrm{~Hz}$ band in the UARS patients $(\mathrm{p}=0.05)$ (fig. 1$)$.

Total sleep. When NREM and REM sleep were not separated, the following absolute powers were computed: delta band (UARS versus OSAS versus controls) $325 \pm 15.25$, versus $221 \pm 13.8$ versus $310.8 \pm 19.3$ $(\mathrm{p}<0.0001)$; alpha band: $309.3 \pm 2.0,191.9 \pm 2.46$, $140.55 \pm 2.55(\mathrm{p}<0.0001)$ (fig. 2$)$. In summary: controls had a significantly higher power density of SWA and significantly less power density in the alpha, beta and sigma bandwidths (ANOVA $\mathrm{p}=0.0001$ ) compared to patients. When UARS and OSAS patients were compared independently to controls, UARS patients also had a significantly higher power density in the alpha and beta bandwidths $(\mathrm{p}=0.001)$ than controls, and overall a similar SWA but the distribution was very different as can be seen in figure 2. SWA is significantly different from controls for each sleep cycle except for cycle 1 . When UARS and OSAS groups were compared to each other, the OSAS group had significantly less power density in the low $(0.5-2 \mathrm{~Hz})$ delta band $(\mathrm{p}=0.05)$, and UARS patients had significantly higher power density in the alpha band $(p=0.01)$. The subanalysis of $1 \mathrm{~Hz}$ increments looking at high theta $(7-8 \mathrm{~Hz})$ and alpha confirmed a significantly higher power density in the 7-8, 8-9 and 9-10 Hz bands $(\mathrm{p}=0.01)$ in UARS versus OSAS patients.

Finally, as mentioned in the nonrapid eye movement sleep section, in UARS, the absolute delta power over the different sleep cycles was significantly different from controls $(p=0.05)$ and from OSAS $(p=0.001)$, respectively. UARS patients did not present the normal decrement in absolute delta power from the first to the last sleep cycle. Also there was always a significantly higher absolute delta power in UARS patients compared to OSAS patients $(\mathrm{p}=0.001)($ fig. 2$)$. The results obtained in the OSHS group were similar to those seen in the OSAS group, with the same significant differences.

\section{Discussion}

The obstuctive sleep hypopnoea syndrome group and the monitoring of airflow

A concerted effort was made to match the subjects very closely, not only for age and sex, but also for BMI. There was not complete success with the sleep hypopnoea syndrome subjects, especially because the definition of "hypopnoea" has been controversial making identification of subjects with pure hypopnoeas difficult. Recently, the use of a nasal cannula with a pressure transducer, to monitor nasal flow has been suggested [13]. As the study was begun with different equipment, it was decided to keep the same apparatus throughout (three out of 12 of the "hypopnoea" subjects had clinical diagnostic studies performed with simultaneous monitoring of airflow using thermocouples and nasal cannulae; these three would have been classified as subjects with OSHS, independent of the recording equipment). The absence of nasal cannulae in the present study is acknowledged, however, information on respiratory effort was obtained by monitoring Poes. During the research monitoring section of the study, "Poes events" were scored, and it is believed that the use of this technique recognizes the breathing disturbances during sleep at least as well as nasal cannulae.

Selection of the population. The selection criteria eliminated obese patients. There were several reasons why patients with this comorbidity were excluded. One of the few investigations on the prevalence of sleep disordered breathing in a representative sample of the general population, and not on a cohort [14], found that most subjects were not obese. As far as clinical populations are concerned, a review of 20,153 patients diagnosed over the years with OSHS with an AHI > 5 events per hour of sleep, with a BMI range $19-71 \mathrm{~kg} \cdot \mathrm{m}^{-2}$, revealed a mean BMI of $25.2 \mathrm{~kg} \cdot \mathrm{m}^{-2}$. The terminology "obstructive sleep apnoea syndrome" 
was selected in the 1970 s to dissociate obese patients with comorbidity from those with absence of such comorbidities and with a problem solely involving the upper airway. Also, the role of obesity as a comorbid factor that may impair the appropriate evaluation of the independent role of the upper airway obstruction during sleep has been emphasized [15]. Obesity has been considered to impact on the evaluation of the independent role of OSAS on the development of hypertension; it clearly impacts on the evaluation of insulin resistance during sleep in patients with sleep disordered breathing [16], and it is a handicap in the investigation of sleepiness related to upper airway dysfunction during sleep. The abnormal insulin resistance and change in glucose metabolism during sleep linked to obesity [16], is an important problem when studying neuronal activity (or its resultant, the sleep EEG). In subjects with upper airway dysfunction, it has been shown that certain obese patients, despite appropriate treatment with nasal continuous positive airway pressure, may present with residual sleepiness and may require prescription of a stimulant [17, 18]. Undoubtedly, some patients with UAR pattern can be obese [19], but the known physiological impact of android obesity on diaphragm position and changes in ventilation/perfusion during REM sleep muscle atonia are factors that led us to select a nonobese population. To eliminate the above bias when comparing sleepiness among the different groups, it was necessary to rigorously match the $\mathrm{BMI}$ of the patients.

Daytime sleepiness and daytime tiredness. The present results are not very different when compared to previous studies [20,21] with MSLT on patients with the OSA polygraphic pattern and variable BMI and AHI. In the present study, there was no significant difference in daytime sleepiness and daytime tiredness between the OSAS and UARS patients. This poses questions raised several times before, though never conclusively answered: what is the mechanism of sleepiness and what is the reason for the lack of correlation between results of the tests or scales of daytime sleepiness and nocturnal findings? Further light is not thrown on this subject. It is not indicated that different EEG patterns identified with spectral analysis of the central leads, were associated with a similar range of daytime results. The similar range of daytime results are seen despite significant differences in the lowest $\mathrm{Sa}_{\mathrm{a}} \mathrm{O}_{2}$ in OSAS and UARS, strictly matched for BMI and age. Why the total sleep time of OSAS patients is not longer i.e. why these patients do not present with "polysomnographic hypersomnia" compared to controls, is of interest. But the total sleep time monitored in the patients in the present study is very much within the range of many reports in the literature. The present research recording was a second night of polygraphic monitoring and subjects became habituated to equipment and techniques. OSAS patients had longer awakenings, falling asleep very quickly when reaching a complete awakening. Why this occurs is unclear. OSAS patients may terminate their apnoeas without clear EEG arousals, but may also stay awake longer. Some of the observed sleep disturbances and longer awakenings are related to nocturnal events such as nocturia. Could obesity be responsible for the variability noted in the speed of falling asleep again or the selected complaint of the subject, i.e. nocturnal sleep disturbance/insomnia versus excessive daytime sleepiness? This is a possibility. The fact that obese patients appropriately treated with verified long term nasal CPAP, may at times, still need to use stimulant medication to stay alert indicates that several factors may be in play in obese subjects with OSAS. It may be of interest to compare comorbid OSAS patients to nonobese patients in order to tease out the independent role of each factor.

Dysmorphia. Morphometric evaluation of the face, using the KusHIDA et al. [7] model indicates that patients with UARS, OSAS and no obesity have mild dysmorphia. Undoubtedly, this index does not indicate if the dysmorphia was similar in all subjects. But it indicates not only should an attempt be made to better define the dysmorphia, but also that the mere presence of the dysmorphia does not explain why there is a different "activation" of the cortex, and different behaviour responses of the upper airway during sleep. It may be helpful in the classification of specific morphotypes, and in the investigation of the role of dysmorphia on the tension on the resting genioglossus muscle during sleep [22, 23].

Sleep analysis. Using RECHTSCHAFFEN and KALES' [9] international criteria, the periods of "awakenings" lasting $\geqslant 30$ s could easily be recognized. OSAS patients had more "awakening" time than any other subjects. These awakenings allow normal breathing for longer periods of time, and occur intermittently. It is not known if it is more detrimental to have repetitive very short sleep disruptions or one longer awakening period. But it is known that longer awakening periods allow the systemic blood pressure to return to normal values, whereas closely related abnormal breathing events are associated with progressive increase in blood pressure with repetitive apnoea [24, 25]. While performing spectral analysis, all arousals, including "awakenings", were removed. This was performed independently of the breathing disorder presented by the subject. Epochs with artefacts were also deleted from the analysis, since sleep is the investigated variable. The sleep EEG from the central leads is clearly different between OSAS and UARS patients. As seen in the different figures, UARS patients have a normal appearance of slow wave sleep, despite the fact that there is less total SWS compared to controls. UARS subjects do not show the usual decline in delta power seen normally from the beginning to the end of the night. They have a higher delta power at the end of the night compared to controls. This absence of clear decline is similar to what is seen in normal subjects submitted to repetitive auditory stimulation with sleep fragmentation [26]. UARS subjects also present a significantly higher amount of low alpha power than other subjects.

The differences in sleep EEG observed between UARS and OSAS/OSHS have led to the following interpretations. Abnormal inspiratory efforts lead to a higher amount of alpha frequency activity in the central EEG leads during sleep in UARS patients. This persistence of higher degree of arousal is associated 
with avoidance of upper airway closure, and the absence of clear blood gas derangement. The development of SWA is also abnormal, with persistence of an abnormally high amount of delta power in the final sleep cycles. Both abnormalities may explain the complaints of daytime sleepiness and/or tiredness in the individual with UARS. In OSAS patients, less alpha power density is observed during the sleep EEG analysis. The predominant NREM sleep frequency is theta with an important decrease in delta power. The absence of high alpha and delta EEG power density during sleep is associated with the complete closure of the airway. There are, thus, clear differences between these two types of sleep EEG, and the development of apnoea and hypopnoea is seen only with one pattern.

Several hypotheses may be proposed to explain the differences in sleep EEG activity seen in OSAS and UARS patients. The differences suggest that both groups of patients have different cortical responses to abnormal respiratory challenges during sleep. There may be a different integration of messages from the periphery (i.e. respiratory apparatus) in the central nervous system.

OSAS patients may have a decreased arousal response as reviewed by BERRY and GLEESON [27], when compared to UARS patients. The decrease in the arousal response would be related to a progressive blunting of reflex responses to abnormal breathing. The chronicity of the breathing problem may lead to a resetting of the receptors involved in the control of upper airway patency during sleep. It has been shown previously [28] that the treatment of OSAS with tracheostomy may result in the progressive resetting of the hypoxic and hypercapnic responses over time. This is in response to the progressively increasing sensitivity of the chemoreceptors, which occurs once sleep fragmentation related to the breathing disorder during sleep has been eliminated. However, hypoxia and hypercapnia are poor arousal stimuli during sleep.

Another possibility is that the sensory message may not be transmitted appropriately in obstructive sleep apnoea syndrome patients. In fact, FRIBERG and coworkers [29-32] have shown in several studies that a local upper airway polyneuropathy can be present in obstructive sleep apnoea syndrome patients. This polyneuropathy is thought to be the result of microtrauma that occurs due to the vibratory effects associated with snoring and possibly those associated with the very negative inspiratory pressure. However, it may be questioned why upper airway resistance patients, especially those who snore, would not develop similar polyneuropathies. An overall significantly different negative oesophageal pressure in the agematched obstructive sleep apnoea syndrome and upper airway resistance patients could not be demonstrated. These hypotheses, however, do not explain completely the differences observed in the sleep electroencephalogram of both types of patients. The authors' interpretation is that upper airway resistance and obstructive sleep apnoea syndrome patients have different responses to mechanoreceptor stimulation. Instead of having different responses due to acquired lesions, the responses may be due to individual genetic build-up. Thus, individuals may present a spectrum of responses, from a very active response to no response. Undoubtedly, environmental factors could worsen the problem, but it is hypothesized that certain individuals, based on their genetic build-up would have difficulty in responding to the challenge during sleep from the start and evolve quickly toward obstructive sleep apnoea syndrome. Their sleep electroencephalogram would show, as noted in the study: 1) the absence of fast reflex responses to mechanoreceptor stimulation; 2) the need to combine different stimuli (hypoxia, possibly shortlived hypercapnia, inspiratory effort) in order to elicit significant electroencephalogram response; and 3) a very different cortical electroencephalogram pattern in the central leads in response to the abnormal breathing. Conversely, upper airway resistance patients would have a very appropriate, exquisite mechanoreceptor response; the message would continuously bombard the brain-stem and would lead to continuous activation of the ascending tracts, through the thalamus to the cortex. These different possibilities of integration of peripheral stimulation could explain the important differences in the sleep electroencephalogram noted in obstructive sleep apnoea syndrome patients compared to upper airway resistance patients. Independent of these possible responses, upper airway resistance and obstructive sleep apnoea syndrome patients present very different sleep electroencephalogram pattern in the central leads. This indicates obstructive sleep apnoea syndrome and upper airway resistance patients cannot be considered similar; they have different brain activity during sleep and this needs to be explained.

\section{References}

1. Guilleminault C, Stoohs R, Clerk A, Cetel M, Maistros P. A cause of excessive daytime sleepiness: the upper airway resistance syndrome. Chest 1993; 104: $781-787$.

2. Lee T, Oh C, Solnick AJ, Guilleminault C, Black JE. Upper airway resistance syndrome and obstructive sleep apnea: a comparative study of clinical features. Sleep Res 1997; 26: 411.

3. Guilleminault C, Kim YD, Stoohs R. The upper airway resistance syndrome. OMF Surg Clin North Am 1995; 7: 243-256.

4. Gould GA, Whyte KF, Rhind GB, et al. The sleep hypopnoea syndrome. Am Rev Resp Dis 1990; 142: $295-300$.

5. Douglass $\mathrm{AB}$, Bornstein $\mathrm{R}$, Nino-Murcia $\mathrm{G}$, et al. Item test-retest reliability of the sleep disorders questionnaire. Sleep Res 1990; 19: 215.

6. Johns MW. Reliability and factor analysis of the Epworth Sleepiness Scale. Sleep 1992; 15: 376-381.

7. Kushida CA, Efron B, Guilleminault C. A predictive morphometric model for the obstructive sleep apnea syndrome. Ann Int Med 1997; 127: 581 - 587.

8. Carskadon MA, Dement WC, Mitler MM, Roth T, Westbrook PR, Keenan S. Guidelines for the multiple sleep latency test (MSLT): a standard measure of sleepiness. Sleep 1986; 9: 519-524.

9. Rechtschaffen A, Kales A, eds. A manual of standardized technology, techniques and scoring system for sleep stages of human subjects. Los Angeles 
(CA). Brain Information Service/Brain Research Institute. UCLA, 1968.

10. American Sleep Disorders Association-Atlas Task Force. Micro-arousals. Sleep 1992; 15: 173-184.

11. Guilleminault $\mathrm{C}$, ed. Sleeping and waking disorders: indications and techniques. Menlo Park, Addison Wesley, 1982; pp.1-435.

12. Feinberg I, Floyd TC. Systematic trends across the night in human sleep cycles. Psychophysiology 1979; 16: $282-291$.

13. Hosselet JJ, Norman RG, Ayappa I, Rappaport DM. Detection of flow limitation with a nasal cannula/ pressure transducer system. Am J Respir Crit Care Med 1998; 157: 1461 - 1467.

14. Ohayon MM, Guilleminault C, Priest RG, Caulet M. Snoring and breathing pauses during sleep: telephone interview survey of a United Kingdom population survey. BMJ 1997; 314: $860-863$.

15. Wright J, Johns R, Watt I, et al. Health effects of obstructive sleep apnoea and the effectiveness of continuous positive airway pressure: a systematic review of the research evidence. BMJ 1997; 314: $851-860$.

16. Stoohs RA, Facchini F, Guilleminault C. Insulin resistance and sleep disordered breathing in healthy humans. Am J Respir Crit Care Med 1996; 154: $170-$ 174.

17. Guilleminault C, Philip P. Tiredness and somnolence despite initial treatment of obstructive sleep apnea syndrome (what to do when an OSAS patient stays hypersomnolent despite treatment). Sleep 1996; 19: Suppl. 9, S117-122.

18. Arnulf I, Homeyer P, Garmal J, Whitelaw WA, Derenne JP. Modafinil in obstructive sleep apneahypopnea syndrome: a pilot study in 6 patients. Respiration 1997; 64: 159-161.

19. Guilleminault C, Kim YD, Palombini L, Li K, Powell $\mathrm{N}$. Upper airway resistance syndrome and its treatment. Sleep 2000; 23: Suppl. 4, 197-207.

20. Roth T, Hartse KM, Zorick F, Conway W. Multiple naps and the evaluation of daytime sleepiness in patients with upper airway sleep apnea. Sleep 1980; 3: 407-424.

21. Guilleminault C, Partinen M, Quera-Salva MA,
Hayes B, Dement WC, Nino-Murcia G. Determinants of daytime sleepiness in obstructive sleep apnea. Chest 1988; 94: 32-37.

22. Rowley J, Permutt S, Willey S, Smith P, Schwartz A. Effect of tracheal and tongue displacement on upper airflow dynamics. J Appl Physiol 1996; 8: $2171-2178$.

23. Odeh M, Schmall R, Gavriely N, Oliven A. Effect of upper airway muscle contraction on supraglottic resistance and stability. Respir Physiol 1993; 92: $139-150$.

24. Tilkian A, Guilleminault C, Schroeder JS, Lerhman KS, Simmons FB, Dement WC. Hemodynamics in sleep induced apnea: studies during wakefulness and sleep. Ann Intern Med 1976; 85: 714-719.

25. Stoohs R, Guilleminault C. Cardio-vascular changes associated with obstructive sleep apnea syndrome. J Appl Physiol 1992; 75: 583-589.

26. Philip P, Stoohs R, Guilleminault C. Sleep fragmentation in normals: a model for sleepiness associated with upper airway resistance syndrome. Sleep 1994; 17: $242-247$.

27. Berry RB, Gleeson K. Respiratory arousal from sleep mechanisms and significance. Sleep 1997; 20: $654-$ 675.

28. Guilleminault C, Cummiskey J. Progressive improvement of apnea and ventilatory response to $\mathrm{CO}_{2}$ following tracheostomy in obstructive apnea syndrome. Am Rev Resp Dis 1982; 126: 14-20.

29. Friberg D. Nerve lesions in pharynx, an aetiology of obstructive sleep apnea. Stockholm, Kongl Carolinska Medico-Chirugiska Institutet, 1997; pp.1-76.

30. Friberg D, Ansved T, Borg K, Carlson-Nordlander B, Larson H, Svanborg E. Histological indication of progressive snorer disease in an upper airway muscle. Am J Respir Crit Care Med 1998; 157: 586-593.

31. Friberg D, Gazecilis B, Hokfelt T, Norlander B. Abnormal afferent nerve endings in the soft palatal mucosa of sleep apnoeics and habitual snorers. Regul Pept 1997; 71: 29-36.

32. Friberg D, Gazelius B, Lindblad LE, Norlander B. Habitual snorers and sleep apnoeics have abnormal vascular reactions of the soft palatal mucosa on afferent nerve stimulation. Laryngoscope 1998; 108: $431-436$. 